\title{
Urinary Tract Infection (UTI) still a Force to be Reckoned with
}

\author{
Raghavendra Rao M.V $V^{1}$.,Kumar Ponnusamy ${ }^{1}$., Sireesha Bala ${ }^{1}$, Sripada Pallavi. $T^{2}$., \\ KrishnaSowmya.M ${ }^{3}$,,Ramanaiah,C.J ${ }^{4}$, Mahendra K.Verma $^{5}$, ,Amin Fateh ${ }^{1,}$ Samir Fatteh $^{1}$ Abraham $^{2}$ \\ Nayakanti1., and Sateesh.Babu. $\mathbf{A}^{\mathbf{1}}$ \\ ${ }^{1}$ Avalon University School of Medicine, Curacao, Central America. \\ ${ }^{2}$ Apollo Institute of Medical Science and Research Institute, Jubilee Hills, Hyderabad, Telangana, \\ ${ }^{3}$ Burjil Hospital, Abu Dhabhi, United Arab Emirates \\ ${ }^{4}$ Amina Hospital Sharjah, United Arab Emirat \\ ${ }^{5}$ Indian Institute of Science Education and Research, Bhopal,India \\ dr.raghavendra@avalonu.org
}

\section{PREPHASE}

Burning pain ,frequent urge to urinate is the first sign of a UTI. It is due to bacteria in the urethra or bladder. Frequent urination is another red flag for an infection. Urine produces peculiar even foul odor and smoky, cloudy. Urine appears pinkish or reddish due to the presence of blood.Young women are commonly infected.Prostate hypertrophy is linked to male disease. It is affecting almost 4 million people in US alone. The chance of UTI is more in women.In females, it affects the bladder and urethra. Women who use diaphragms, spermicidal agents are more at risk. Menopause women are more vulnerable to infection. Infection of upper urinary tract,consisting of the kidney and pelvis ,is known as pyelonephritis.Infection of the lower tract may involve the bladder (Cystitis), urethra (Urethritis) or prostate (Prostatitis) Intercourse is common association of UTI. Catheters increase the risk.Obstruction of urinary flow increase the risk.Bacterial adherence favors persistence.Fever is usually absent.Enterobacteriaceae and gram positive bacteria appear with complications.Back and perirectal pain are the signs of UTI.Pyuria suggests UTI but not specific.Chronic disease is the source of cystitis.Kidney infection results in permanent kidney damage. Take plenty of water/fluids to flush out bacteria.Wipe front to back. This helps the spread of bacteria from the anus into the bladder. Decreased estrogen levels during menopause cause changes that make the urinary tract more susceptible to bacteria. Most patients with UTI have uncomplicated cystitis, which is one of the most common infections in the United States, especially in sexually active women. Escherichia coli is the most common cause of urinary tract infection. Staphylococcus saprophyticus is a frequent cause of cystitis in women, probably related to its occurrence as a part of normal vaginal flora. Klebsiella, Enterobacter, Proteus, and Serratia are the primary opportunistic and often nosocomial pathogens. Pseudomonas aeruginosa is an opportunistic pathogen and a major cause of hospital-acquired infections.

Keywords: Escherichia coli ,Klebsiella, Pseudomonas aeruginosa, Enterobacteriaceae, uncomplicated cystitis, , cefaclor, 


\section{Introduction}

Invasion of rectal bacteria by direct extension or by lymphogenous or hematogenous spread may also constitute other possible routes.(1)Due to several anatomical and hormonal changes, pregnant women are more susceptible to develop Urinary tract infections (UTI) (2). UTI is a major health problem, it has been reported among $20 \%$ of the pregnant women and it is the most common cause of admission in obstetrical wards.(3)

Although various microorganisms can cause UTI, Escherichia coli is the most common cause of disease in $80 \%-90 \%$ of cases. $(4,5)$

Staphylococcus saprophyticus as a second agent is a distant second to E.coli,causing 5 to $10 \%$ of infections.S.saprophyticus presents as a more aggressive disease with more approximately one half of the patients showing involvement in the upper urinary tract. (6) Urinary tract infections also account for up to $40 \%$ of nosocomial infections catheters these hospital acquired infections tend to be more serious because the bacteria resistant to drug treatment and patients are often in poor general health.(7)

Clinical characteristics, etiology and antimicrobial susceptibility patterns may differ from country to country.(8)

Antibiotics are administered only if gas formation is localized in the renal pelvis and there is no invasion in the kidney parenchyma. .(9). During urinary tract infections, invading bacteria may either promote or prevent host cell death by interfering with cell death pathways.(10)

Uncomplicated urinary tract infections (UTIS) are common in otherwise healthy individuals. Half of all women will get one or more UTIs before reaching their mid-30s, and recurrent infections are frequent also in women without any anatomical abnormalities in the urinary tract $(11,12)$

Urinary tract infections (UTIS) are a severe public health problem and are caused by a range of pathogens, but most commonly by Escherichia coli, Klebsiella Pneumoniae, Proteus mirabilis, Enterococcus faecalis andisand Staphylococcus saprophyticus..(13)

As the most common bacterial infection that requires medical care, UTIs vary greatly by clinical presentation and therapeutic management. Urinary tract infections affect a variety of patients with different biological and procedural risk factors(e.g., age, sex, pregnancy, catheters and urologic interventions). However, not all bacteria require antibiotic therapy, particularly in the presence of ASB. Antibiotic stewardship practices are essential to promote judicious antibiotic use for UTIs. This can significantly reduce antibiotic resistance because UTIs are the most common infections leading to an antibiotic prescription.(14)

Procalcitonin (PCT) as a potential biomarker that can help in differentiating between lower UTI and pyelonephritis in the pediatric age group.(15)

Uropathogenic Escherichia coli is the causative agent for $>80 \%$ of uncomplicated urinary tract infections (UTIS). Uropathogenic E. coli strains express a number of virulence and fitness factors that allow successful colonization of the mammalian bladder.(16)

,MAPK activators, and lymphocyte signaling molecules.(17) 
Raghavendra Rao M.V., Kumar Ponnusamy., Sireesha Bala, Sripada Pallavi.T, KrishnaSowmya.M., Ramanaiah,C.J.,Mahendra K.Verma, Amin Fateh, Samir Fatteh Abraham Nayakanti, and Sateesh. Babu. A; Urinary Tract Infection (UTI) still a Force to be Reckoned with. Journal of Biomedical Engineering and Medical Imaging, Volume 5, No 4, August (2018), pp 23-29

Diseases Group of the French Pediatric Society set up an active surveillance network in pediatric centers across France in 2014.(18)

Clear instructions for the interpretation of urine cultures by the laboratory technicians are indispensable to obtain standardized, reliable, and clinically useful results.(19)

Congenital abnormalities of the kidney and urinary tract have a high prevalence (3.5-43\% in pediatric population.(20)

\section{History}

It was described by the Egyptians as "sending forth heat from the bladder.(22) Effective treatment did not occur until the development and availability of antibiotics in the 1930s before which time herbs, bloodletting and rest were recommended

Urinary tract infections have been described since ancient times. The first written description, found in the Ebers Papyrus, dates to around the 1550 BC.(23)The Egyptians described a urinary tract infection as "sending forth heat from the bladder(24)

Herbs, bloodletting, and rest were the common treatments until the 1930s, when antibiotics became available.(23)

\section{Significant Gap in Research}

In most cases, UTIs can be diagnosed just from the symptoms and there is no need for laboratory testing.. The urine is tested for urinary nitrites, white blood cells (leukocytes), or leukocyte esterase.. However, women with negative cultures can still improve with antibiotic treatment (25) UTI symptoms in old people can be vague, and diagnosis can be difficult as there is no really reliable test (26) It is a common urological condition. Sometimes it is impossible to eradicate it because of the development of drug-resistant bacteria. So the wrong therapy is likely to make sensitive organism resistant to drugs. Hence prior isolation of causative organisms and their sensitivity to antimicrobial drugs should be done before any rational treatment is given to the patient.(27)

Staphylococcus saprophyticus is a frequent cause of cystitis in women, probably related to its occurrence as a part of normal vaginal flora. Klebsiella, Enterobacter, Proteus, and Serratia are the primary opportunistic and often nosocomial pathogens. Pseudomonas aeruginosa is an opportunistic pathogen and a major cause of hospital-acquired infections such as UTI, particularly in patients who have been subjected to catheterization, instrumentation, surgery, or renal transplantation or to prior antibiotic therapy.(28)

\section{Major Advances and Discoveries}

Infected children, men, and those who experience UTI relapse should be investigated with intravenous pyelography to allow detection and correction of any factor causing predisposition to infection.(29)The risk of UTI, both cystitis, and pyelonephritis, can be increased by several factors, especially sexual intercourse, particularly with a new sexual partner. Immunodeficiency and urogenital tract anatomical abnormalities have been considered the essential risk factors for recurrent UTI. In healthy women,Voiding dysfunction and behavioral factors also increase the risk of recurrent UTI.Sexual intercourse and estrogen deficiency in postmenopausal women might have the strongest association 
with recurrent UTI.. Vaccines for recurrent UTI are recommended by the latest guidelines and are available on the market.(30)

Recent research has revealed many novel concepts in recurrent UTI including pathogenesis, risk factors, biomarkers, and prevention. Nowadays recurrent UTI may be considered a distinct disease and patients with recurrent UTI should be managed aggressively.

\section{Ideas where the Research go Next?}

Together, these mechanisms work in concert to help eradicate a UTI. In all likelihood, these mechanisms are constantly being utilized by our urinary tract to ward off invading pathogens without a single symptom or invasive infection.(31)

UTI are the some of the most common bacterial infections, resulting in billions of dollars in health care annually (32)

The only effective treatment option available-antibiotics $(33,34)$ These are considered complicated UTIs, defined as those in the presence of factors that predispose to persistent or relapsing infection, such as foreign bodies (calculi, indwelling catheters), obstruction, renal failure, and urinary retention.(35)

Initial therapy is based on the local susceptibility patterns of $E$. coli and other uropathogens. For the treatment of cystitis, an adequate urinary antibiotic concentration is important to ensure response to therapy Nitrofurantoin is recommended for the treatment of cystitis. It is highly active against $E$. coli, with $0.9 \%$ resistance among female outpatients.Trimethoprim/sulfamethoxazole remains a highly effective agent for the treatment of uncomplicated cystitis, with cure rates of $90 \%-$ $100 \%$.Fluoroquinolones (e.g., levofloxacin or ciprofloxacin) are recommended for the treatment of uncomplicated pyelonephritis

\section{Current Debate}

Fosfomycin trometamol has in vitro activity against most Enterobacteriaceae spp. including ESBLproducing isolates and Enterococcus spp.Studies of $\beta$-lactam antibiotics (e.g., amoxicillin/clavulanate, cefaclor, cefdinir, cefpodoxime, and ceftriaxone) report lower efficacy than with fluoroquinolones and trimethoprim/sulfamethoxazole.(36) Depending on the susceptibility of isolated strains, different oral relay possibilities were available: $30 \%$ of isolates were susceptible to cotrimoxazole, $50 \%$ were susceptible to ciprofloxacine and only $37 \%$ were resistant to both antibiotics, which led to the prescription of a non-orthodox combination.(37). Thus urine culture should be performed as screening and diagnostic tool of UTI in pregnancy in this setting.(38) UTIs vary greatly by clinical presentation and therapeutic management.Urinary tract infections affect a variety of patients with different biological and procedural risk factors (e.g., age, sex, pregnancy, catheters and urologic interventions). However, not all bacteriurias require antibiotic therapy, particularly in the presence of ASB. Antibiotic stewardship practices are essential to promote judicious antibiotic use for UTIs. This can significantly reduce antibiotic resistance because UTIs are the most common infections leading to an antibiotic prescription.(39)

Foreign bodies (calculi, indwelling catheters), obstruction, renal failure, and urinary retention. (40) 
Raghavendra Rao M.V., Kumar Ponnusamy., Sireesha Bala, Sripada Pallavi.T, KrishnaSowmya.M., Ramanaiah,C.J.,Mahendra K.Verma, Amin Fateh, Samir Fatteh Abraham Nayakanti, and Sateesh. Babu. A; Urinary Tract Infection (UTI) still a Force to be Reckoned with. Journal of Biomedical Engineering and Medical Imaging, Volume 5, No 4, August (2018), pp 23-29

\section{REFERENCES}

[1] Domingue, G. J. \&Hellstrom, W. J. G. (1998). Prostatitis. Clin Microbiol Rev 11, 604-613.

[2] Dafnis E, Sabatini S: The effect of pregnancy on renal function: physiology and pathophysiology. Am J Med Sci 1992, 303(3):184-205.

[3] Bacak SJ, Callaghan WM, Dietz PM, Crouse C: Pregnancy-associated hospitalizations in the United States, 1999-2000. Am J ObstetGynecol2005, 192(2):592-597.

[4] Elder JS. Urinary tract infections. In: Kliegman RM, Stanton BF, St.Geme JW III, Schor NF, Behrman RE, editors. Nelson textbook of pediatrics. 20th ed. Philadelphia (PA): Elsevier Saunders, 2016:2554-63.

[5] Wald ER. Cystitis and pyelonephritis. In: Feigin RD, Cherry J, Demmler-Harrison GL, Kaplan SL, editors. Feigin and Cherry's text book of infectious diseases. 6th ed. Philadelphia (PA): Elsevier Saunders, 2009:554-69.

[6] Agace, W., Hedges, S., Andersson, U. et at.. (1993). Selective cytokine production by epithelial cells following exposure to Escherichia coll.Infection and Immunity ,61, (2), 602-609.

[7] Al Mamun, A. A. M., Tominaga, A. and Enomoto, M. (1996). Detection and characterization of the flagellar master operon in the four Shigella subgroups. Journal of Bacteriology 178: 3722-3726.

[8] Cheol-In Kang, Jieun Kim, Dae Won Park, Baek-Nam Kim, U-Syn Ha, Seung-Ju Lee,JeongKyun Yeo, Seung Ki Min, Heeyoung Lee, and Seong-HeonWie.Clinical Practice Guidelines for the Antibiotic Treatmentof Community-Acquired Urinary Tract Infections. Infect Chemother 2018;50(1):67100 .

[9] Aboumarzouk OM, Hughes O, Narahari K, Coulthard R, Kynaston H, Chlosta P, Somani B. Emphysematous pyelonephritis:Time for a management plan with an evidence-basedapproach. Arab J Urol 2014;12:106115.

[10] Diego Martin-Sanchez, Miguel Fontecha-Barriuso, Maria Dolores Sanchez-Niño, Adrian M. Ramos, Ramiro Cabello, Carmen Gonzalez-Enguita, Andreas Linkermann, Ana BelénSanz, and Alberto Ortiz.Cell deathbased approaches in treatmentof the urinary tract-associated diseases: afight for survival in the killing fields. Martin-Sanchez et al. Cell Death and Disease, 2018, 9118, 1-14.

[11] Hooton, T. M. 2001. Recurrent urinary tract infection in women. Int. J. Antimicrob.Agents 17: 259-268.

[12] Foxman, B. 1990. Recurring urinary tract infection: incidence and risk factors.Am. J. Public Health 80: 331333.

[13] Ana L. Flores-Mireles, Jennifer N. Walker, Michael Caparon, and Scott J. Hultgren.

[14] Urinary tract infections: epidemiology, mechanisms of infection and treatment options. Nat Rev Microbiol. 2015 May ; 13(5): 269-284. 
[15] Helen S. Leeand Jennifer Le. Urinary Tract Infections. PSAP 2018 Book 1,Infectious Diseases, 7-28.

[16] Neha Nanda and ManishaJuthani-Mehta. Novel Biomarkers for the Diagnosis of Urinary TractInfection-a Systematic Review. Biomarker Insights 2009:4 111-121.

[17] Kelsey E. Sivick, Matthew A. Schaller, Sara N. Smith andHarry L. T. Mobley. The Innate Immune Response toUropathogenicEscherichia coli InvolvesIL-17A in a Murine Model of Urinary TractInfection. J Immunol2010; 184:2065-2075.

[18] Benjamin L. Duell, Alison J. Carey, Chee K. Tan, XiangqinCui, Richard I. Webb, MakrinaTotsika, Mark A. Schembri,Petra Derrington, Helen Irving-Rodgers, Andrew J. Brooks,Allan W. Cripps, Michael Crowley and Glen C. Ulett. Innate Transcriptional Networks Activated inBladder in Response to UropathogenicEscherichia coli Drive Diverse BiologicalPathways and Rapid Synthesis of IL-10 forDefense against Bacterial Urinary TractInfection. J Immuno/2012; 188:781-792.

[19] FouadMadhiet al, 2018.Febrile urinary-tract infection due toextended-spectrum beta-lactamaseproducing Enterobacteriaceae in children: AFrench prospective multicenter study. PLoS One. 2018 Jan 25;13(1):e0190910.

[20] MatthijsOyaert, Britt Van Meensel, ReinoudCartuyvels, Johan Frans, WimLaffut, Patricia Vandecandelaere, Hans De Beenhouwer.Laboratory diagnosis of urinary tract infections: Towards a BILULUconsensus guideline. Journal of Microbiological Methods 146 (2018), 92-99.

[21] Song R and Yosypiv I V 2011. Genetics of congenital anomalies of the kidney and urinary tractPediatr. Nephrol. 26(3),353-64.

[22] Al-Achi, Antoine (2008). An introduction to botanical medicines: history, science, uses, and dangers. Westport, Conn.: Praeger Publishers. p. 126. ISBN 978-0-313-35009-2. Archivedfrom the original on 201605-28

[23] Wilson...], [general ed.: Graham (1990). Topley and Wilson's Principles of bacteriology, virology and immunity: in 4 volumes (8. ed.). London: Arnold. p. 198. ISBN 0-7131-4591-9.

[24] Al-Achi, Antoine (2008). An introduction to botanical medicines: history, science, uses, and dangers. Westport, Conn.: Praeger Publishers. p. 126. ISBN 9780313350092.

[25] Wilson...], general ed.: Graham (1990). Topley and Wilson's Principles of bacteriology, virology and immunity: in 4 volumes (8. ed. ed.). London: Arnold. p. 198. ISBN 0713145919.

[26] Nicolle LE (2008). "Uncomplicated urinary tract infection in adults including uncomplicated pyelonephritis". Urol Clin North Am 35 (1): 1-12, v. doi:10.1016/i.ucl.2007.09.004. PMID 18061019

[27] Woodford, HJ; George, J (2011 Feb). "Diagnosis and management of urinary infections in older people.". Clinical medicine (London, England) 11 (1): 80-3. PMID 21404794 
Raghavendra Rao M.V., Kumar Ponnusamy., Sireesha Bala, Sripada Pallavi.T, KrishnaSowmya.M., Ramanaiah,C.J.,Mahendra K.Verma, Amin Fateh, Samir Fatteh Abraham Nayakanti, and Sateesh. Babu. A; Urinary Tract Infection (UTI) still a Force to be Reckoned with. Journal of Biomedical Engineering and Medical Imaging, Volume 5, No 4, August (2018), pp 23-29

[28] The short textbook of Medical Microbiology,Satish Gupte, Ninth Edition, Jaypee Brothers, Medical Publishers(p) LTD PP-454

[29] Lippincott's illustrated reviews,Richard A.Harvey,Pamela.C.Champe,Microbiology second Edition,PP374375.

[30] Sherris Medical Microbiology, fifth Ed,Kenneth .J.Ryan,C.George Ray,Nafees ahmad,W.Lawrence Drew, James.J.Plorde, Mcgraw Hill-Medical,pp-939-941

[31] Jia-Fong Jhang, Hann-ChorngKuo.Recent advances in recurrent urinary tract infection from pathogenesis and biomarkers to prevention. Tzu Chi Medical Journal 2017; 29(3): 131-137.

[32] John David Spencer, Andrew L. Schwaderer,Brian Becknell,Joshua Watson, and David S. Hains. The innate immune response during urinary tract infection and Pyelonephritis. PediatrNephrol. 2014 July ; 29(7): 1139-1149.

[33] StammWE,NorrbySR,Urinary tract infections:Disease panorama and changes.J.of.Inf.disease ,2001:183,Suppl,:si,s4 (pubmed)

[34] Chen YH,Ko.WC,Hsueh PR,Emerging resistance problems and future perspectives in pharmacotherapy for complicated urinary tract infections.Expert Opin Pharmacother:2013,14:587-596

[35] Kostakioti M,Hultgren SJ,Hadjifrangiskou,M.Molecular blue print of ureopathogenic Escherichia coli virulence provides clues toward the development of anti virulence therapeutics. Virulence 2012:3:592594(pubmed)

[36] Levison ME, Kaye D. Treatment of complicated urinary tract infections with an emphasis on drug-resistant gram-negative uropathogens. Curr Infect Dis Rep. 2013;15(2):109-115.

[37] Helen S. Lee and Jennifer Le. Urinary Tract Infections. PSAP 2018 Book 1.Infectious Diseases, 7-28.

[38] Fouad Madhi et al. Febrile urinary-tract infection due to extended-spectrum beta-lactamase-producing Enterobacteriaceae in children: A French prospective multicenter study. PLoS One. 2018 Jan 25;13(1): e0190910.

[39] Hamdan Z Hamdan, Abdel Haliem M Ziad, Salah K Ali, Ishag Adam. Epidemiology of urinary tract infections and antibiotics sensitivity among pregnant women at Khartoum North Hospital. Annals of Clinical Microbiology and Antimicrobials 2011, 10:2, 1-5.

[40] Sanchez GV, Babiker A, Master RN, et al. Antibiotic resistance among urinary isolates from female outpatients in the United States in 2003 and 2012. Antimicrob Agents Chemother 2016;5:2680-3.

[41] Levison ME, Kaye D. Treatment of complicated urinary tract infections with an emphasis on drug-resistant gram-negative uropathogens. Curr Infect Dis Rep. 2013;15(2):109-115. 